\title{
Synchronous manifestation of 160-min pulsations of the ground pressure and Z-component of geomagnetic field at Moscow, Apatity, Oulu, Yakutsk and Tixie
}

\author{
V. Ye. Timofeev ${ }^{1}$, D. G. Baishev ${ }^{1}$, L. I. Miroshnichenko ${ }^{2}$, \\ S. N. Samsonov ${ }^{1}$ and N. G. Skryabin ${ }^{1}$ \\ ${ }^{1}$ Yu.G. Shafer Institute of Cosmophysical Research and Aeronomy, SB RAS, \\ 677980, Yakutsk, Russia \\ email: timofeev@ikfia.ysn.ru \\ ${ }^{2}$ N.V. Pushkov Institute of Terrestrial Magnetism, Ionosphere \\ and Radio Wave Propagation (IZMIRAN), RAS, \\ Troitsk, Moscow Region, Russia
}

\begin{abstract}
Oscillations of the ground pressure and Z-component of geomagnetic field with a period of 160-min in December 2003 and March 2004 have been studied by regular measurements carried out over the territory of Europe and Siberia. As the analysis has shown, in December 2003 these specific oscillations are manifested synchronously from Europe up to Siberia, preferentially in the form of 'packages' ('quanta') consisting of two-five pulses. Their average amplitudes in December 2003 are of $0.0115 \pm 0.0023 \mathrm{mb}$ in the ground pressure and of $0.323 \pm 0.070 \mathrm{nT}$ in the Z-component. We suppose that the 160-min pulsations in those both terrestrial parameters represent the effect of a gravitational wave of the same period coming from the Galaxy center.
\end{abstract}

Keywords. Earth, Sun: activity, gravitational waves

\section{Introduction}

Gravitational waves in the Universe can be formed only by very dense bodies of major mass (so-called relativistic objects (see review by Rudenco 2006). Their nearest and strongest concentration is related to the central regions of our Galaxy. According to Kotov \& Kotov (1996) the 160-min pulsations of the Universe can benefit the gravitational wave radiation when interacting with such objects.

If there exists a specific, preferred arrival direction of the gravitational waves from the central parts of the Galaxy (in particular, with a period of 160-min) then it should be manifested at the Earth by changes of the wave phase in a sidereal time when the Earth moves along its orbit around the Sun. In this case, one can expect some differences in the manifestations of pulsations between major areas in the meridian and latitudinal sections depending on the arrival direction of gravitational wave to the Earth. It may take place, for example, due to the gravitational wave changes its specific parameters (dimension, polarization etc.) that are still unknown.

To register gravitational waves it is suggested to use the planet Earth as a whole as a unique very massive device. We assume that this device is constructed by the analogy of heavy cylinder described by Rudenco (2006), with the only difference in that the central part of the cylinder is substituted by the mass of the whole Earth planet, and the edges of it - by the whole Earth's atmosphere. The gravitational wave covers the whole such a 'cylinder' instantly and completely. As a result, the pressure of terrestrial atmosphere 
will have the same phase on the major areas of the planet. One can register synchronous changes of the pressure using the system of pressure sensors distributed over the globe. Simple calculations show that the sensitivity of such 'device' will be greater by a factor of $10^{16}$ than it is specified by Rudenco (2006). By this analogy it is possible to construct a 'device' in which the central part of 'cylinder' will be a core of the Earth, and its edges the rest terrestrial parts. The registration is carried out, in particular, by magnetometers that are operating synchronously and are distributed all over the terrestrial globe.

\section{Processing and analysis of experimental data}

As a preliminary, a raw observational data have been processed twice by a band probability filter with a band of oscillation periods from 40 to 180 minutes. Fig.1a shows the results of processing of 5-min values of the ground pressure using the superposed epoch technique in two positions of the Earth at its orbit, December 2003 and March 2004, at 5 stations (Tixie, Apatity, Oulu, Yakutsk, and Moscow). A zero meridian (December 1 at 00:00 UT) has been accepted as a reference point of superposition. In Fig.1b we preesent the results of processing of 1-minute values of Z-component for the same periods at Oulu, Moscow, Novosibirsk, Irkutsk, and Lvov. It may be seen from the Figures 1a,b that the manifestations of 160-min pulsations depend on sidereal time.

In Fig.1a (solid lines) one can see that in the ground pressure all phases obtained from the December data coincide at all stations all over the territory of Europe and Siberia. This figure shows also that a synchronism in March is worse manifested than in December (in March it is seen only at two stations - Tixie and Moscow). It means that there takes place a distinction in the phases of synchronism manifestation of 160-min pulsations in March and December, presumably because of the distinction of sidereal and solar time. In March the synchronism is kept only for the latitudes of Moscow and for the polar latitudes regions (for example, Tixie).

In the geomagnetic field, we deal with the response to some effects in the current systems existing at large depths and in the Earth's core, such effects being modulate a magnetic field at the terrestrial surface. Mechanisms of formation of such response are still unknown. But in December the synchronism takes place at all stations (see Fig.1b, solid lines). In March there is a change of phases but their character is manifested in another way than in the ground pressure. The effects calculated by a superposed epoch technique, on the average, are equal to $0.0115 \pm 0.0023 \mathrm{mb}$ in the ground pressure and to $0.323 \pm 0.070 \mathrm{nT}$ in the Z-component in December.

\section{Some implications of the results}

Since we suppose that pulsations are caused by the gravitational wave which instantly covers the whole terrestrial globe then, after taking the sum of the filtered data of ground pressure in December such pulsations at all stations will have the identical phases. Really, after such summation, the 160-min pulsations in December are manifested in the form of 'packages' of 2-5 pulses.

For any 'package', in the most cases, the distribution of amplitudes is characterized by a gradient (force) to the direction of the process development (i.e., to the direction of general course of time). If so, the 'packages' under the influence of such forces 'fly' to the future, apparently, with acceleration. It is not unlikely that this feature may be one of the experimental signatures of accelerated expansion of the Universe found out recently.

From the temporal change of average amplitude of 160-min pulsations in the ground pressure, another one effect seems to become apparent, this effect being caused by some 


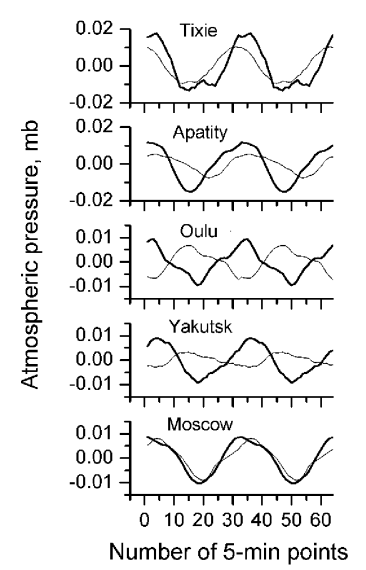

a)

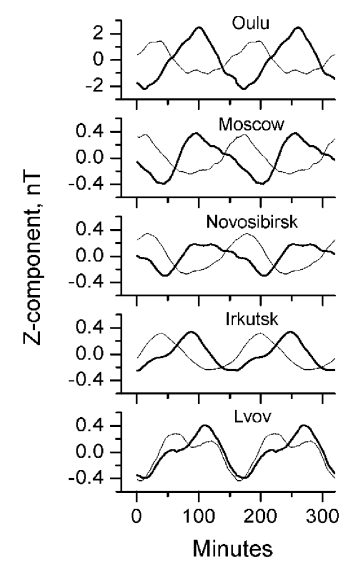

b)

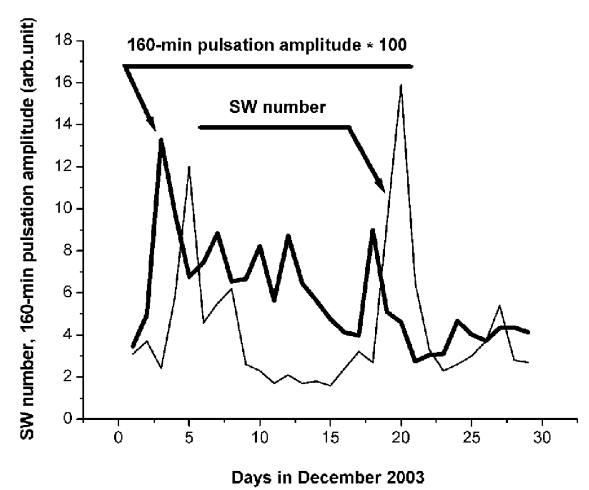

c)

Figure 1. a) Average temporal changes of 160-min pulsations on the interval of two periods (in December 2003 - solid lines, and in March 2004 - thin lines) in the ground pressure; b) Z-component of geomagnetic field at the territory of Europe and Siberia; c) Comparison of variations of 160-min pulsation amplitude (solid line) and solar wind number density (thin line).

non-stationary processes in the Galaxy center that amplify the generation of gravitational waves. There are some grounds to expect that solar response to gravitational waves is amplified inside the Sun in the form of a sound wave. About 1 hour after, it manifests itself at the Sun 'surface' as an increase in the solar wind density and temperature. In the vicinities of the Earth those changes of density and temperature will manifest themselves after $\sim 2$ days since the solar wind speed is relatively small (on the average, it is about $440 \mathrm{~km} / \mathrm{s}$, but can be more or less). By this time, the response to such processes in the center of Galaxy has already taken place on the Earth. Proposed effect of 2-4 days advance of solar activity in the ground pressure may be seen in Fig.1c. In fact, in the time profile of average amplitude of the 160-min pulsations in the ground pressure, one 'burst' in the pressure was observed on December 3 and in the solar wind - on December 5 ; the second burst was fixed on December 16 and 20, respectively. By their general view, the bursts in the ground pressure repeat, even in details, the bursts of solar wind density.

No telescope can observe what has happened at those moments in the center of our Galaxy because proposed effects are caused by changes of intensity of just gravitational waves, but not optical and any electromagnetic radiation. Such phenomena are very rare events (apparently, about one per year), but they have supposedly caused two bursts in solar wind density on December 5 and 20, 2003.

\section{References}

Kotov, V. A. \& Kotov, S. V. 1996, Radiophysics and Quantum Electronics, 39, 807

Rudenco, V. N. 2006, Earth and Universe (in Russian), 6, 28 